AperTO - Archivio Istituzionale Open Access dell'Università di Torino

\title{
VDML4RS: a tool for reputation systems modeling and design
}

\section{This is a pre print version of the following article:}

Original Citation:

\section{Availability:}

This version is available http://hdl.handle.net/2318/1637104

since 2017-05-22T11:21:34Z

Publisher:

ACM

Published version:

DOI:10.1145/2993283.2993286

Terms of use:

Open Access

Anyone can freely access the full text of works made available as "Open Access". Works made available under a Creative Commons license can be used according to the terms and conditions of said license. Use of all other works requires consent of the right holder (author or publisher) if not exempted from copyright protection by the applicable law. 
This is the author's final version of the contribution published as:

Bettini, Lorenzo; Capecchi, Sara. VDML4RS: a tool for reputation systems modeling and design, in: SSE 2016 Proceedings of the 8th International Workshop on Social Software Engineering, ACM, 2016, 9781450343978, pp: 8-14.

The publisher's version is available at:

http://dl.acm.org/ft_gateway.cfm?id=2993286\&amp;ftid=1808277\&amp;dwn=1

When citing, please refer to the published version.

Link to this full text:

http://hdl.handle.net/2318/1637104 


\section{VDML4RS: a tool for reputation systems modeling and design}

\author{
Lorenzo Bettini \\ Dipartimento di Statistica, Informatica, \\ Applicazioni Università di Firenze, \\ Viale Morgagni, 59 - 50134 Firenze, Italy \\ lorenzo.bettini@unifi.it
}

\author{
Sara Capecchi \\ Dipartimento di Informatica Università di Torino, \\ corso Svizzera 185, 10149 Torino, Italy \\ sara.capecchi@unito.it
}

\begin{abstract}
Reputation has a great influence on interactions between system providers and users (e.g. in e-commerce). Despite that, reputation systems implemented so far show many deficiencies and suffer from reliability problems. One of the causes is the lack of appropriate software engineering methodologies dedicated to requirements collection and design of reputation systems. On the other hand, business process modeling, service design and reputation system modeling share many needs (represent a variety of entities involved in the related domains), aims (improve services/business in order to meet the user's needs) and problems (they all involve stakeholders with very different backgrounds and skills). Starting from the intuition above, this paper proposes VDML4RS a tool for conceptual modeling of reputation systems. The aim is to provide a tool to represent reputation requirements of the system-to-be from the very beginning in the software development allowing mutual understanding between requirements engineers and stakeholders avoiding the socio-technical mismatch in communication that can delay and harm the development.
\end{abstract}

\section{CCS Concepts}

-Software and its engineering $\rightarrow$ Requirements analysis; Open source model; Programming teams;

\section{Keywords}

Reputation systems, conceptual modeling, business model analysis, VDML

\section{INTRODUCTION}

Research problem. The reputation of an entity is used to make a value judgement and defines the expectation of other entities about its behaviour starting from information (observations, data, facts) of its past behaviour. Reputation has a great influence on interactions between system providers and users (e.g. in e-commerce). Despite that, as outlined in [2, 25, 4], existing reputation systems show many deficiencies: i) lack of connection between reputation statements and its context, e.g judgement about product, delivery, price, interaction with seller is melted in a 5 star claim type plus a detailed written feedback; ii) incomplete or non-comprehensive provided information which causes incorrect perception of the service reputation by the user; iii) no distinction between expressions of fact and opinion, that is between objective and subjective claims. This deficiency deeply influences their credibility and usefulness.

Moreover, in spite of public administrations' efforts to improve citizen participation through the use of new technologies, reputation system evaluating public services (schools, hospitals, administrations) are almost absent.

The above issues could be addressed in two ways: by collecting feedbacks about different aspects of products/services in order to elaborate and display comments and ratings in structured formats; by exploiting sources of objective data that can give important information about products and services quality. For instance subjective feedbacks about schools could be integrated with information about the meaning of quality certifications (guarantees about food provenance in canteen service, cleaning standards) or with Open Data. Indeed quality control and guarantee organizations control the quality of services to ensure products are designed and produced in order to fit customer expectations; open data collected and stored by Open Governments contain information that are relevant to evaluate public services quality. This information are not exploited because they are neither easily reachable nor represented in a clear way.

As a matter of fact both the two sources of reputation data about a service (standardization/public organizations data and users ratings) should be integrated in order to build a more articulated reputation since they are related to different aspects of the value delivered to users. Summarizing, current reputation systems can be improved concerning what is being shared and how it is being shared: reputation has a subjective nature but it can be also built by providing a certain degree of objectivity and automation by analysing and collecting data related to some part of the service. The aim of our research are: $(i)$ to improve the structure of users feedbacks in order to clearly connect ratings and comments to specific part of services/products; ( $i i)$ to integrate subjective feedbacks with objective data: this data must be analysed and elaborated in order to be displayed to users in a clear mode; (iii) to connect ratings and comments about specific aspects of the delivered value to the roles responsible for creating that value.

Research solution. One of the main causes of the deficiencies described above is the lack of support for reputation systems development, starting from the very early stages, that is, domain analysis and requirements collection. So far reputation models have been added after-the-fact in an ad-hoc perspective, limiting re-usability and suffering scalability problems. The entities that play a role in a reputation system (i.e. users, services, products, transactions), 
should be expressed and modelled at a level that enables mutual understanding between requirements engineers and stakeholders so as to avoid the socio-technical mismatch in communication that can delay and harm the system development: requirements analysis is one of the most important phase of software development. At this stage many problems arise because technical issues considerations have to be taken into account together with social and organizational ones: there is need for conceptual models and tools that are closer to the domain (roles, values) instead of the information system/program structure (classes, methods) [7]. The aim of a conceptual model for reputation system is to model those features that compose the value delivered to/perceived by users and costumers.

Instead of proposing (another) new conceptual model we looked for an existing model to extend in order to exploit the research performed so far on conceptual modeling. Since the aim is to model those features that compose the value delivered to and perceived by the customers/users we considered conceptual models for service design and business modeling. We started from the idea that the design of a reputation system shares, with business process modeling and service design, many needs, aims and problems: (i) to represent the variety of entities involved in the related domains. In the case of reputation systems in essential to model all the aspects that compose the value of a product/service; (ii) to improve services/business in order to meet the user's needs. For reputation systems is important to link feedbacks related to different aspects of the service to the responsible roles; (iii) to overcome the sociotechnical mismatch due to the different background and skills of involved stakeholders.

The concepts, the roles, the objectives, the phases of business modeling and service design should be strictly connected to those of the design of a reputation system used to evaluate a business or a service: a business model reflects management's hypothesis about what customers want, how they want it, what they will pay for it and how an enterprise can organize itself to best meet customer needs [22]. Business modeling simplifies how an organization structures all its activities and resources to develop the right value proposition for the right clients and finally make money from all these processes; service design is user-centred and involves different actors and different customer groups, employee and interfaces [21]. Understanding the nature of relations between people and organizations, between business partners of different kinds is now understood to be central to design services. The problems in this setting are related to the analysis and design that are tightly related to human and organizational activities focusing for instance on the identification of a set of dependencies among roles involved in service/product delivery.

Current status of work and contribution. From this starting point in [8] we analysed the extension of models for service design and business conceptual representation towards reputation systems design. We proposed an extension of the Value Delivery Modelling Language (VDML) [5], a modeling language for business innovation and towards the representation of concepts and requirements for reputation systems.

VDML abstracts the organization structure of an enterprise, the creation and exchange of value, the capabilities that produce that value, the management of resources, people and roles, interactions with business partners: it provides the right abstractions to represent the concepts related to reputation systems in the design phase when roles and entities must be clearly detected: reputation sources and target roles (service user, service provider, certification service third party), parts of the service that can be objectively and subjectively evaluated etc. VDML is under development as an OMG (Object Management Group [1]) standard.
In this paper we describe VDML4RS a set of visual tools (diagram editors and tables) that can be used to capture requirements, design and specify desired properties of reputation systems. The starting point for the tool implementation is the model proposed in [8]. To develop VDML4RS we extended the VDML meta-model that we built with the Eclipse Modelling Framework (EMF) [20]. The aims of VDML4RS is to allow mutual understanding between reputation system committees and designers filling the gap between requirements of reputation systems, expressed at a social/business level and the design of ratings/feedbacks and entities' reputation which has to be modelled at technical level. The use of VDML4RS should ease the detection of: 1) those aspects that compose the value of the service delivered to users/customers, 2) the information that come from objective data (es. delivery time, performance measurement, certification on products provenance and production methods), 3) the roles responsible for producing the value. The proposed solution follows Social Software principles: simplicity, community-orientation and quick collaboration in order to involve larger groups of stakeholders to collaborate in requirements definition. On the other hand, sufficient formality must be provided in order to serve typical demands of requirements engineering, such as structured access or efficient analysis and post-processing of the collected requirements. VDML4RS has been designed for early phases of requirements engineering with many distributed stakeholders collaborating informally and to ease participation instead of providing advanced and complex features. It should emphasize the social experience of developing requirements for a reputation system for stakeholders. In order to test the adequacy of the proposal we used the VDML4RS diagrams to develop a website collecting feedbacks and certified information about kids nurseries (see section 7). We gathered feedback from two kind of end-users: requirements designers and domain experts (nurseries staff).

The rest of the paper is organised as follows: Section 2 resumes problems related to reputation systems and analyses requirements for reputation systems conceptual modeling; Section 3 presents the main features of our tool using a use case; Section 4 provides some details about the tool implementation; Section 5 describes the results of a preliminary evaluation among VDML4RS users; Section 6 discusses related works; Section 7 concludes the paper and lists our current and future works.

\section{PROBLEMS AND MODELING REQUI- REMENTS}

The terminology used in this section is taken from [10] where basic objects involved in a reputation system are described. First reputation statements have the following structure: a source makes a claim about a target. A source is any entity that can make a reputation claim (users, third party certifications etc.). A claim is the value that the source assigns to the target. Claims have a type and a value. We do abstract from different types of claims (quantitative vs qualitative, raw vs normalised) and we just distinguish between claims coming from a subjective opinion and those coming from an objective measurement: for instance a claim about payment service of an e-store could come from subjective comments of customers or from objective data (percentage of information leaks, delay in payment procedures). A target is any reputable entity that can be the object of a reputation claim. Let us consider the following reputation statement: user $U$ rates 3 stars over 5 product $P$; user $U$ is the source, 5 star is the claim type, 3 star is the claim value, product $\mathrm{P}$ is the target. In order to overcome the problems outlined in the introduction an adequate modelling language should be able to represent: source and target roles, target entities that can be 


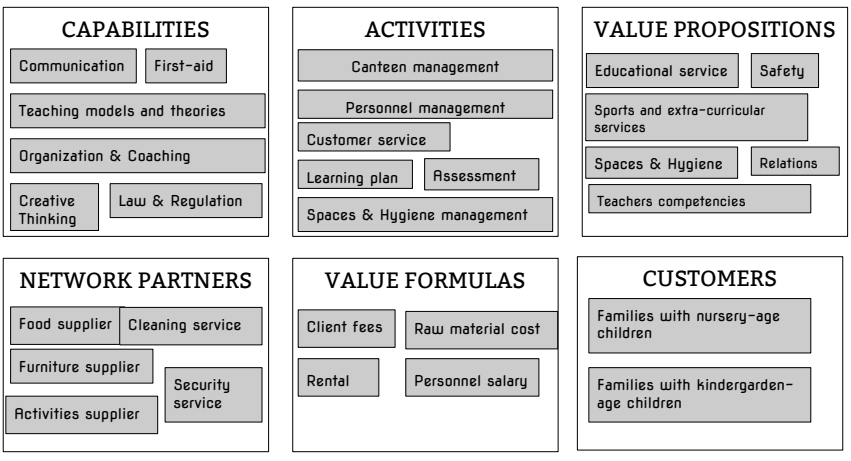

Figure 1: Nursery cube model

subjectively and objectively measured, the complete multiplicity of targets that compose an overall service/value, the link between targets of claims and roles in the business service responsible for them (each part of a service that is evaluated should be directly and easily connected with roles and actors responsible for it in the business organisation).

To address the above requirements we need models describing the activities, competences, resources and skills that are strictly linked and connected to create the value delivered by the organization to customers, that is, a business model.

To support the creation of highly complex businesses that deal with volatile technologies new procedures for creating and testing business models have emerged. One of these developments is the CUBE model proposed in [16], a conceptual instrument that helps the definition of 1) customers 2) value proposition 3) value formula 4) network partners 5) capabilities 6) activities. Customers represent that segment of buyer interested in the value proposition of the organization; value proposition is the value perceived by target customers of the product and service offering; value formula is a realistic view of the sources of revenue and cost; network partners represent all the voluntarily initiated cooperative agreement between two or more companies in order to create value for the customer; capabilities are the competencies necessary that should be created in house and contribute to the power of a business model; activities are all the operations to put in action value proposition. We illustrate our proposal by using an example, a web platform that collects reviews of schools to allow parents to orient themselves within the educational offer and choose the appropriate school for their children. Figure 1 describes the cube model associated with our case study. The value proposition of the school is centred on the quality of proposed services, spaces, relationship, and competencies. Customers are clustered by the need of clear and structured information about educational services and the use of the Internet as a source of information. The capabilities are centred on all those activities that help to maintain the quality of service and the users experience. The network is based on supplier partnership which represents the cost of the value formula. Value Delivery Modelling Language supports the six views of the cube with dedicated diagrams. In the next section we introduce VDML4RS a tool to collect requirements of reputation systems associated to specific business/services through the use of VDML diagrams (extended towards reputation systems conceptual modeling).

\section{VDML4RS}

We start with Value exchange proposition diagram which depicts roles and exchanged products and services expressed as value propositions. The value proposition exchange diagram related to our example is illustrated in Figure 2 where roles are represented by circles and value propositions by squares. Value proposition is composed by eight elements divided into two groups: 1) first group: it is the closest to the customer perception and composed by educational service, canteen, spaces, interaction with families; 2) second group: it is the closest to the partners and it is composed by food, activities, cleaning and security devices. These elements depend on out-house partnership expertise. Standard plain arrows go from provider roles to value propositions (i.e. from food supplier to food) and from value propositions to recipient roles (i.e. from food to nursery). In order to represent the abstractions needed to model reputation systems requirements VDML concepts has been extended in VDML4RS by providing roles and value proposition specialisations and new relations between them.

A source role is any participant who can make a claim about a target and is identified by a light grey circle. In our example source roles are customers who can express claims about any aspect of the nursery, food supplier who can certificate provenance and quality of the delivered food, supplier of extra activities who provides data about the usefulness of the proposed courses. A target role is any participant that is the object of a reputation claim and it is represented with black circle. In our example the target of the reputation system is the nursery.

The Value propositions are the ideal target of reputation claims: source roles provide their judgements, data and measurements about products and services that compose value propositions. Targets of reputation statements can be evaluated with objective data and measurements or with subjective comments/ratings. Then, value propositions can be target of objective, subjective claims or both. We denote them with squares with three different kinds of colours depicted in Figure 2: i) food, activities, educational competencies, educational services, canteen and spaces can be evaluated objectively (from providers/certification companies collecting data) and subjectively (from users); ii) cleaning can be evaluated subjectively by users.

Relations. Dashed-dot arrows represent a connection between a source role and its objectively measured target; in our example they connect: i) the certification company to spaces and canteen since it certifies spaces safety (room/person, security devices, emergency exits) and food preparation (kitchen/tools adequacy, food conservation); ii) food supplier provides evidences about provenance and ingredients; iii) extra activities providers can demonstrate the skills of their staff by providing documents about their training; iv) open data provided by public administration can show important information about public nurseries concerning requirements for both spaces and teachers training. For the sake of readability in Figure 2 we do not represent the relation between source and target of a subjective claim (the graphical element is a dashed arrow going from the customer to food, activities, educational competencies, educational services, canteen, spaces, cleaning).

The analysis performed so far can be summarised in Figure 3 where each source role is associated with the objectively or subjectively evaluated value propositions and the role responsible for creating it: certification company provides data about canteen and spaces. The responsible role for these two value proposition is the nursery: all these data are shown in the table "Target Objective Value Propositions of Source Role certification company". These table are automatically created in VDML4RS from each value proposition diagram. 


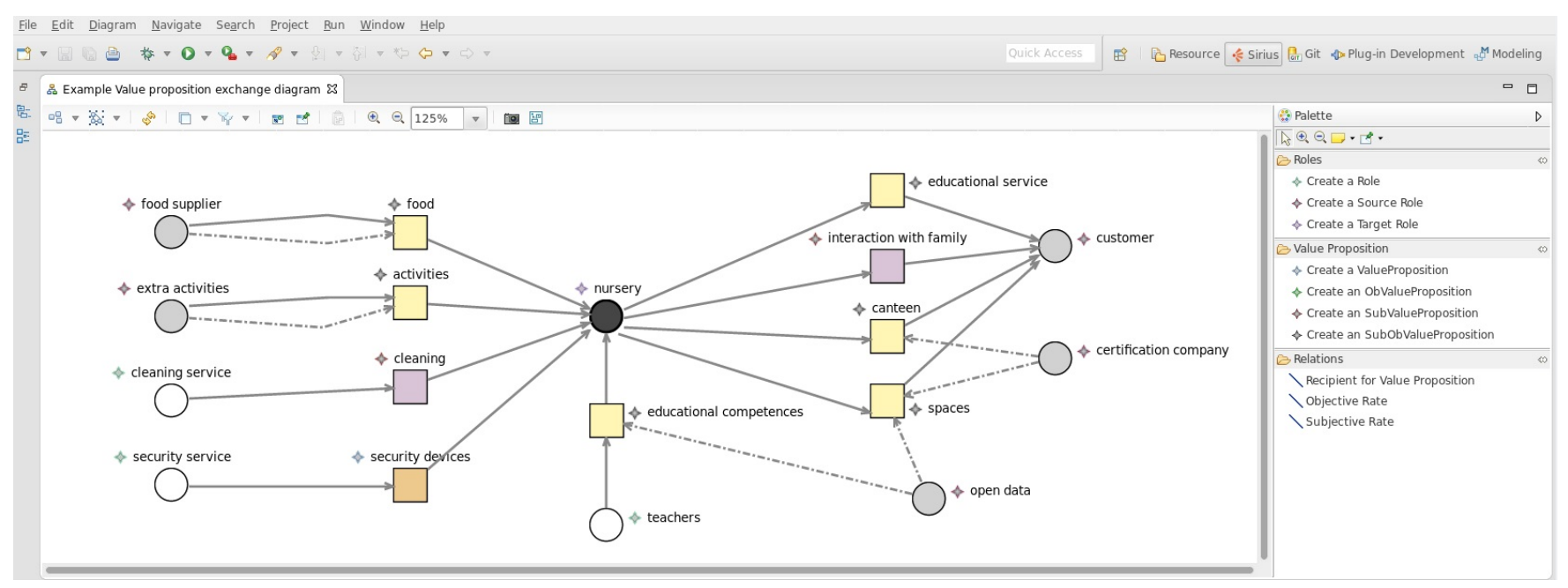

Figure 2: Value proposition exchange diagram of nursery

\begin{tabular}{|c|c|c|c|c|}
\hline \multicolumn{3}{|c|}{ 国 Target Objective Value Propositions of Source Role certification company $\mathfrak{Z}$} & \multicolumn{2}{|c|}{ 困 Target Subjective Value Propositions of Source Role customer $\mathbb{\&}$} \\
\hline & \multicolumn{2}{|l|}{ Responsible Role } & & Responsible Role \\
\hline$\diamond$ canteen & \multicolumn{2}{|l|}{ Target Role nursery } & $\diamond$ activities & Source Role extra activities \\
\hline$\diamond$ spaces & \multicolumn{2}{|l|}{ Target Role nursery } & $\diamond$ canteen & Target Role nursery \\
\hline \multicolumn{3}{|c|}{ W Target Objective Value Propositions of Source Role food supplier $\llbracket$} & $\diamond$ educational competences & Role teachers \\
\hline \multicolumn{3}{|c|}{ Responsible Role } & $\diamond$ food & Source Role food suppler \\
\hline \multicolumn{3}{|c|}{ Source Role food supplier } & $\diamond$ spaces & Target Role nursery \\
\hline & & & $\diamond$ cleaning & Role deaning service \\
\hline \multicolumn{3}{|c|}{ W Target Objective Value Propositions of Source Role open data $₹ 3$} & $\diamond$ interaction with family & Target Role nursery \\
\hline & & Responsible Role & & \\
\hline \multicolumn{2}{|c|}{$\diamond$ educational competences } & Role teachers & & \\
\hline \multicolumn{2}{|l|}{$\diamond$ spaces } & Target Role nursery & & \\
\hline
\end{tabular}

Figure 3: Summary of source roles-subjective/objective evaluated value propositions and the role responsible for creating it.

All the graphical elements and the relations between them can be added or removed through a dedicated menu (see Section 4). These tables can be used by developers and nursery managers to reason about the shape of the reputation system associated to the service: what are the objective information that can/should be displayed to customers? What are the fields of a feedback form that customers should fill when rating their experience? The result of the proposed methodology for reputation system requirements analysis is a complete set of subjective and objective targets: i) objective claims can be used to structure and display data provided by standardisations/certifications in order to make their meaning clearer for users ii) subjective claims can be used to design surveys for customers feedback collection.

The second diagram that can be created in our tool is inspired by VDML activity diagrams which describe the typical flow of activities which a participant, service provider and other stakeholders perform in a service execution. Figure 4 illustrates the daily experience of families in nurseries. A nursery service user begins his experience at the school entrance, evaluating hygiene and safety aspects of space. Then the welcome time, that involves teacher, as educational activities, meal involves the staff canteen, and extracurricular activities may involve external personnel specialized in individual activities. This diagram shows the roles responsible for creation and management of the value propositions proposed to the customer and can be used to further refine the design of feedback surveys since it describes the complete experience of a customer while using the service.

Using the above diagrams the connection between reputation targets, value proposition and the roles responsible for producing it will provide information needed by managers on how to improve activity and operations, understand their impact on the rest of the business and reorganise collaborations between internal units and departments and with external business partners. This will be possible by exploiting the features of VDML model which relates all the parts of the business cube.

\section{VDML4RS IMPLEMENTATION}

The VDML4RS implementation consists of several parts. First, we implemented the VDML model in EMF [20]. Then, the original VDML was extended with the new elements described in the paper (i.e., source/target roles and subjective/objective value propositions). Finally, we implemented several Eclipse graphical editors and graphical views using Sirius [11].

The main graphical editor is the "Value proposition exchange diagram" shown in Figure 2, which has already been described in the previous section. This is effectively an editor since it allows to modify the underlying VDML model: besides moving and removing existing relations (represented by arrows), the editor provides a toolbar (in Figure 2 it is the one shown on the right) that allows to add new elements (roles and value propositions) and relations. 


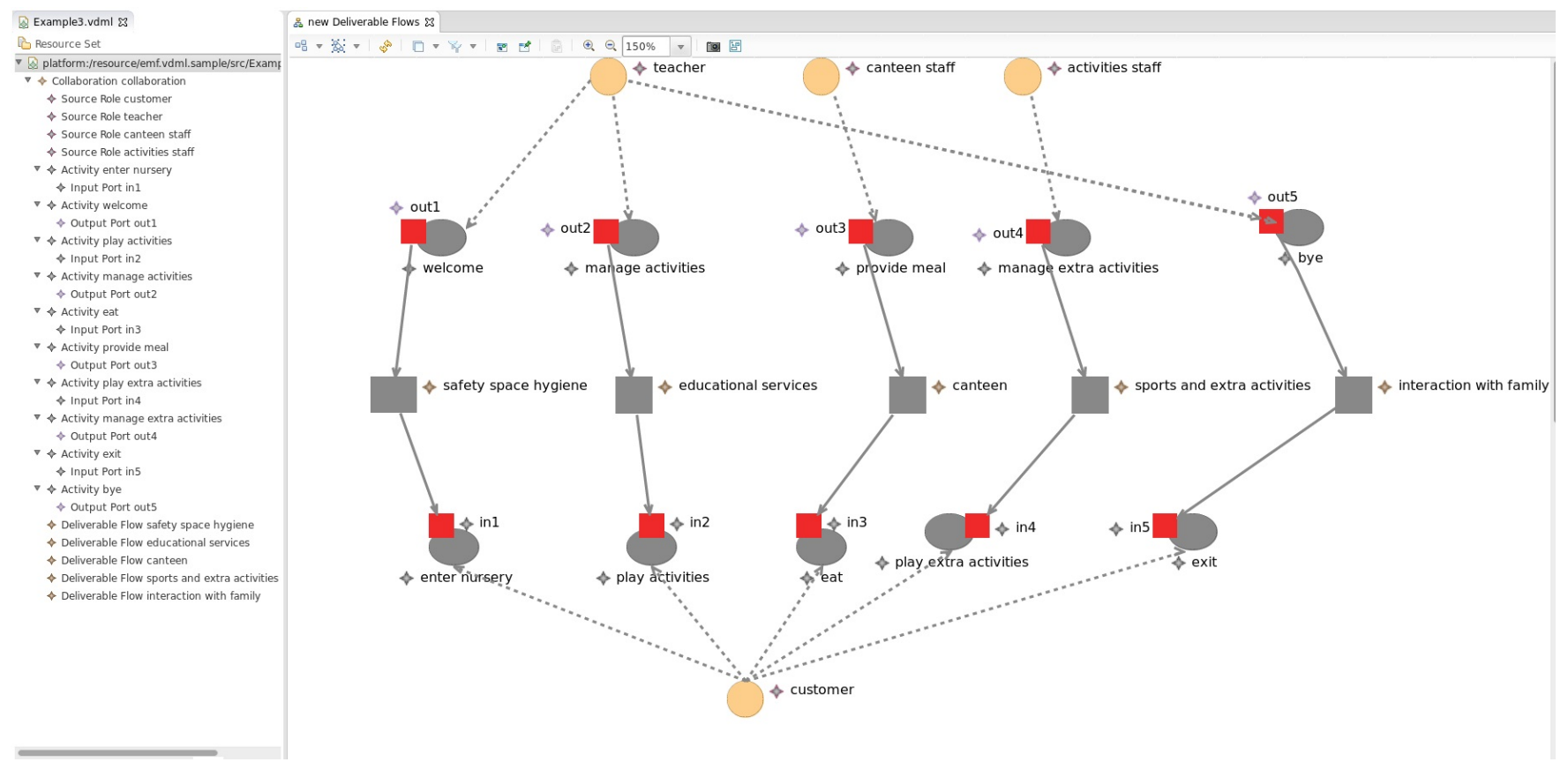

Figure 4: Diagram of customer's daily activities in the nursery

Similarly for the editor shown in Figure 4; in the Figure we also show the underlying EMF model that is represented in the editor. The tables shown in the paper are not editable, since they aim to provide a different view on the model, in a tabular form. We might extend such tables to be editable as well in the future, but we think that a diagram editor is more suitable for modifying the model visually.

Our VDML4RS Eclipse plug-in is open source software available at https://github.com/LorenzoBettini/vdml4rs. As a future work, it would be interesting to provide also a textual representation of the VDML model; with that respect, we plan to investigate its implementation using Xtext [6], the de-facto standard Eclipse framework for the development of programming languages and domain-specific languages (DSLs).

\section{VDML4RS EVALUATION}

In order to test the adequacy of the proposal we have recently started to gather feedback from two kinds of end-users: requirements designers and domain experts. We proposed VDML4RS diagrams for the requirements collection, design and development of a website collecting feedbacks and certified information about kids nurseries. Some of the diagrams and tables produced during the development of this use case has been shown in Section 3. This use case is very useful to test VDML4RS for many reasons: 1) in this context the value propositions that are delivered to users and that can be evaluated by them in a reputation system are very complex and peculiar. There are many aspects that have to be considered when evaluating a service for kids, these aspects are hard to understand by outsiders of the specific domain (requirements analysts); 2) there are examples of information about the service coming from quality certifications 3 ) the problem of socio-technical mismatch between requirement analysts and domain experts (nurseries staff and owners) is relevant in this case.

The use of the diagrams proposed in VDML4RS allowed mutual understanding between requirements engineers and stakehold- ers avoiding the problems due to communication mismatch. According to [9] there are several decisions to select relevant information concerning reputation targets:

- what actions are relevant for reputation? VDML4RS diagrams and table allow coarse grained and fine grained detection of the action that are relevant for target's reputation;

- how to obtain information about these actions? the analysis performed on the use case shows how to decide whether collecting information by asking users' feedback or storing objective data;

- how to aggregate/display information? we showed how to structure reputation according to the variety of different aspects that compose service/business value perception by user; according to [26] data alone does not create transparency. Analysis and visualisation are required to describe the relationship between data, feedbacks and context.

Following [23] VDML4RS has been evaluated focusing on the following points: modelling language usability, detection of missing concepts, usability of graphical representation, concepts' semantics clarity. The usability criteria used in or evaluation are: effectiveness which measures how users are able to achieve their specified goals through design, efficiency which focuses on the effort made by users to learn and use the modelling language, finally satisfaction checks the overall impression collection feedbacks by users.

Since the very early stages of the platform creation, we involved end-users of the modelling language in evaluation activities. Users are all stakeholders who interacted with the modelling language and tool: two programmers, two software analysts, around 20 stakeholders from nurseries' staff/owners/managers. Following [23] users' experience was gathered by the use of forms and by observing their interaction during the use of the model. In the following we summarize the results gathered in the users' answers and from external observation: 
- Participants noted that the modelling language is quite complex for people who are not experts in business models. This issue confirms the difficulties due to the socio-technical mismatch. Programmers and software analysts needed to correctly model all the aspects related to the delivery of value proposition and network business collaboration of educational services. For this purpose a good training for the modelling language was an essential prerequisite. Involved programmers were also asked to try an alternative way to collect requirements by using UML. Although more familiar with UML in the end they concluded that, once the concepts related to business modeling and value delivering are clear, the interaction with educational staff was much easier using VDML. This confirm the need for conceptual models and tools that are closer to the domain (roles, values) instead of the information system/program structure.

- Staff from nursery asked for a clearer comparison between objective and subjective claims related to the same target value proposition.

- The modelling effort to provide nurseries with insight regarding the connection between user satisfaction and the enterprise organisations and responsibilities was highly appreciated by nurseries managers and owners since this analysis is expected to both improve outcomes and reduce the cost of services.

The evaluation of VDML4RS has greatly helped us in identifying problems to be considered in the current development of the tool. We found that a few concepts are missing but there are several usability issues concerning the representation of concepts specific to business modelling and value delivery. In the following we summarize benefits of the proposed approach for different stakeholders categories.

Reputation system designers, software analysts. The new proposed methodology should encourage the design of users surveys in such a way that the criteria that make the service reputation can be modelled clearly and rated separately so that the evaluation of an entity is no longer generalized. Each value can be related to a certain context therefore giving the opportunity of easier storage and elaboration of feedback to improve services. More specifically the benefits for developers could be listed as: 1) consistent terminology, 2) appropriate levels of abstraction facilitating the development of the model in several domains and ensuring the simplicity and reusability of the embedded information, 3) support for identifying the appropriate stakeholders, users and relevant sources of information.

Customers/users, firms/organizations. Linking customer feedback as part of an appropriate reputation systems to specific business model elements can easily improve the organization's output in line with customers' needs and features. The advantages of this model are for both organizations and customers. Concerning organization, a reputation model that focuses on the the business model could improve the value of organizations: firms can detect the problematics and modify or eventually delete the causes of the bad reputation; a positive side effect could be the efficiency improvement related to cost decrease and to reward increase.

\section{RELATED WORKS}

There are lot of proposals in the literature addressing the design and the development of reputation systems.

Methodologies for reputation systems design and implementation. We started from [10], [4] and [9] for a review on reputation systems problems, design requirements and methodology description.
Conceptual modelling. The VDML language approach is based on a unification of concepts from a number of business modeling approaches like REA (Resource Event Agent) [12], e3Value [13] and the concept of role collaboration modeling also used in SoaML [19]. We refer to [5] for a comparison with these approaches. We decided to start from VDML since it provided all the set of modeling features that we needed to minimize an extension towards reputation systems al so from a technical point of view since it is an OMG standard metamodel. [17] extends UML towards trust and reputation concepts while [3] reviews the literature on ontologies for reputation systems; finally Reputation Object Model [2] is a conceptual model identifying relevant concepts related to reputation systems. Some of the concepts represented in these works can be easily mapped to ours. On the other hand, central to our proposal, is the representation of business concepts coming from the original VDML that allow the connection between reputation targets, value proposition and the roles responsible for producing it in order to provide information needed by managers on how to improve activity and operations, understand their impact on the rest of the business and reorganise collaborations between internal units and departments and with external business partners.

We do not address issues related to reputation claims measurements and computing functions since, so far, we have addressed the problem of modeling reputation objects. A future step in this direction is discussed in Section 7.

Frameworks. [24] presents a framework for the analysis of reputation systems describing the requirements for the development of reliable reputation systems and the features necessary to achieve them. The use of our tool in the design phase facilitates the realization of reputation systems addressing many requirements listed in [24]: ratings and reputation should discriminate user behaviour, reputation should be assessed using a sufficient amount of information, the reputation system should differentiate reputation information by the interaction it represents, reputation should capture the evolution of user behaviour. [18] proposes a framework to help developers in implementing reputation systems for cloud applications while [15] implements a SaaS reputation service to develop customizable reputation systems. These frameworks are oriented toward implementation of reputation systems while our proposal belongs in the requirements phase so it can be used in a preliminary phase where all stakeholders are involved.

In the literature many reputation models have been proposed based on interactions among agents. It is out of the scope of the paper to analyse them. We refer to [14] for a survey on the subject. Among all the proposals, TROPOS [7] shares with us the goal for a socio-technical approach for requirements engineering in context where there are stakeholders with different goals and backgrounds.

\section{CONCLUSION AND FUTURE WORKS}

In this paper we proposed a tool to support the representation of reputation management requirements by exploiting tools and models for service design and business modeling. The outcomes can be used both in the initial stage to detect and model reputation sources and surveys for feedbacks and a later stage when evaluating feedbacks results since in every reputation claim the target is connected with the role responsible for creating the associated value.

Concerning future works we plan to extend and refine the current implementation in many directions. First both the subjective evaluations expressed by customers and objective data can be measured and stored with associated values in order to analyse and compare business' performance, improvements and bottlenecks. In the VDML value propositions are measurable benefits delivered to a recipient in association with a deliverable that would influence the 


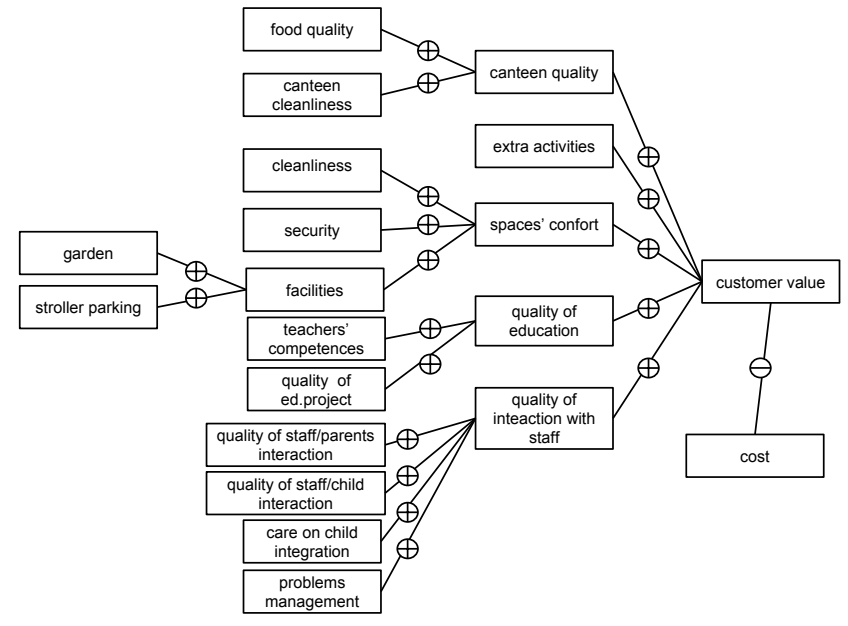

Figure 5: Measure graph of the online store values

desirability of a service. VDML supports value measurement and the computations to assess the impact of performance of specific capabilities on the cost, quality of end services.

While modelling activities, the analyst can build a VDML measurement dependency graph like that in Figure 5. The plus (+) and minus (-) signs on the arcs indicate if the source measurement increases or decreases the target measurement. The graph depicts the aggregations of value measurements that model satisfaction levels of the value proposition.

Indeed, VDML incorporates the SMM (Structured Metrics Metamodel) specification to represent the measurement libraries and the measurable properties of model element: in SMM, a measure is a method that is applied to characterize an attribute of something by assigning a comparable quantification or qualification. We plan to exploit these features to built measurement dependency graph to depict the aggregations of value measurements that model satisfaction levels of the value proposition. Another important feature is the direct representation of claims' type and a value (e.g. 5 stars, 5 likes). This feature will be included in the tool by extending the tables summarizing reputation sources and targets.

\section{ACKNOWLEDGMENTS}

This work has been partially supported by European Union's Horizon 2020 project HyVar (grant agreement No. 644298), by ICT COST Action IC1402 ARVI and by Ateneo-CSP project RunVar (D16D15000360005).

\section{REFERENCES}

[1] Object Management Group. http://www.omg.org/.

[2] R. Alnemr, S. Koenig, T. Eymann, and C. Meinel. Enabling usage control through reputation objects: A discussion on e-commerce and the internet of services environments. $J$. Theor. Appl. Electron. Commer. Res., 5(2):59-76, Aug. 2010.

[3] R. Alnemr and C. Meinel. From reputation models and systems to reputation ontologies. In IFIPTM, pages 98-116. Springer, 2011.

[4] R. Alnemr and C. Meinel. Why rating is not enough: A study on online reputation systems. In CollaborateCom, pages 415-421, 2011.

[5] A. J. Berre, Y. Lew, B. Elvesaeter, and H. de Man. Service Innovation and Service Realisation with VDML and
ServiceML. In EDOCW, pages 104-113. IEEE, 2013.

[6] L. Bettini. Implementing Domain-Specific Languages with Xtext and Xtend. Packt Publishing, 2nd edition, 2016.

[7] V. Bryl, P. Giorgini, and J. Mylopoulos. Designing socio-technical systems: From stakeholder goals to social networks. Requirement Engineering, 2009.

[8] S. Capecchi and P. Pisano. Reputation by design: using VDML and ServiceML for reputation systems modeling. In ICEBE, pages 191-198. IEEE, 2014.

[9] C. Dellarocas. Designing reputation systems for the social web. SSRN Electronic Journal, 2010.

[10] R. Farmer and B. Glass. Building Web Reputation Systems. Yahoo! Press, USA, 1st edition, 2010.

[11] E. Foundation. Sirius, 2015. http://eclipse.org/sirius.

[12] G. L. Geerts and W. E. McCarthy. An ontological analysis of the economic primitives of the extended-REA enterprise information architecture. International Journal of Accounting Information Systems, 3:1-16, 2002.

[13] J. Gordijn and J. Akkermans. Value-based requirements engineering: exploring innovative e-commerce ideas. Requirements Engineering, 8(2):114-134, 2003.

[14] H. H. He Lijian and Z. Wei. A survey of trust and reputation systems in multi agent systems. Journal of Computer Research and Development, 45(7), 2008.

[15] C. Hillebrand and M. Coetzee. The design of a configurable reputation service. In TrustBus, pages 60-70. Springer, 2015.

[16] P. Lindgren and O. H. Rasmussen. The Business Model Cube. Journal of Multi Business Model Innovation and technology, 2013. 3rd edition.

[17] F. Moyano, C. Fernandez, and J. Lopez. Towards engineering trust-aware future internet systems. In Advanced Information Systems Engineering Workshops, volume 148 of LNCS, pages 490-501. Springer, 2013.

[18] F. Moyano, C. Fernandez-Gago, and J. Lopez. A framework for enabling trust requirements in social cloud applications. Requir. Eng., 18(4):321-341, Nov. 2013.

[19] OMG. Service oriented architecture Modeling Language (SoaML) Specification, Version 1.0.1. OMG Document formal/2012-05-10, 2012.

[20] D. Steinberg, F. Budinsky, M. Paternostro, and E. Merks. EMF: Eclipse Modeling Framework 2.0. Addison-Wesley Professional, 2nd edition, 2009.

[21] M. Stickdorn, J. Schneider, and K. Andrews. This is service design thinking: Basics, tools, cases. Wiley, 2011.

[22] D. J. Teece. Business models, business strategy and innovation. Long Range Planning, 43(2-3):172 - 194, 2010. Business Models.

[23] S. Trösterer, E. Beck, F. Dalpiaz, E. Paja, P. Giorgini, and M. Tscheligi. Formative user-centered evaluation of security modeling: Results from a case study. IJSSE, 3(1):1-19, 2012.

[24] S. Vavilis, M. Petkovic, and N. Zannone. A reference model for reputation systems. Decision Support Systems, 61:147 154, 2014.

[25] Y. Yao, S. Ruohomaa, and F. Xu. Addressing common vulnerabilities of reputation systems for electronic commerce. J. Theor. Appl. Electron. Commer. Res., 7(1):1-20, Apr. 2012.

[26] S. Yelp. 3 an inquiry into effective reputation and rating systems. The Reputation Society: How Online Opinions are Reshaping the Offline World, page 25. 